\title{
Understanding the Macroscopy of Malignant Lesions of the Female Internal Genital Tract
}

\author{
Josenel Maria Barcelos Marçal ${ }^{* 1}$, Rafael Nazário Bringhentti ${ }^{2}$, Rita de Cássia Sant'Anna Alves ${ }^{1}$, Adriana Vial Roehe ${ }^{1}$, Fernanda \\ Correa Pinto ${ }^{3}$, Judite Tais Silveira Fagundes", Péttala Rigon ${ }^{3}$, Shala Wesner Martins, and Giuseppe Dick Bonato ${ }^{6}$ \\ ${ }^{1}$ Department of Pathology and Legal Medicine, Federal University of Health Sciences of Porto Alegre, Brazil \\ 2School of medicine of Pontifícia Universidade Católica do Rio Grande do Sul, Porto Alegre, Brazil \\ ${ }^{3}$ Resident physician of the Santa Casa de Misericórdia Hospital, Porto Alegre, Brazil. \\ ${ }^{4}$ Biologist at Santa Casa de Misericórdia Hospital, Porto Alegre, Brazil \\ ${ }^{5}$ Biologist at Santa Casa de Misericórdia Hospital, Porto Alegre, Brazil \\ ${ }^{6}$ Student of the medical course, Federal University of Health Sciences of Porto Alegre, Brazil
}

${ }^{\star}$ Corresponding author: Josenel Maria Barcelos Marçal, Department of Pathology and Legal Medicine, Federal University of Health Sciences of Porto Alegre, Rua Sarmento Leite 245, Porto Alegre, RS, 90050170, Brazil; E-mail: josenel.copetti@gmail.com

Received: February 08, 2019; Accepted: February 19, 2019; Published: February 26, 2019;

\begin{abstract}
Communication and agreement between pathologists and gynecologists and oncologists and others experts is important for a good understanding of patient management and further diagnostic decision and complementary surgical conduct. On some occasions, there may be doubts about reports or even diagnoses from a transoperative examination. Hence, we deem an interdisciplinary action to be important, where we can explain the diagnostic procedures of pathology in order to elucidate doubts and review basic principles of the macroscopy of genital folds. We describe step by step, in an uncomplicated way, the macroscopic examination of the female internal genital tract.
\end{abstract}

Keywords: Macroscopy, Female Internal Genital Tract, Neoplasms

\section{Introduction}

With previous diagnosis of neoplasms the uterine body (adenocarcinoma) or uterine cervix (epidermoid carcinoma), ovaries, uterine tubes and vagina are examined by pathologists is of fundamental importance to the prognostic assessment and therapy.

Many gynecologists, oncologists, radiotherapists and other specialist have doubts about the data described in an anatomopathological report. Therefore, we listed a few steps in the macroscopy routine of a pathological anatomy laboratory in an attempt to explain the data supplied in the reports.

\section{Hysterectomy}

The first step in the macroscopic examination of a hysterectomy is the opening of the uterus for fixation, with the introduction of a probe, The weight of the organ without the adnexa, which should be assessed separately; the measurements of the uterus, such as height (from the bottom to the cervix) and latero-lateral and anteroposterior diameter; and measurements of the cervix (height, diameter of the cervix and diameter of the external (orifice) are checked. The thickness of the myometrium, endometrium and endocervix are also checked. If the piece presents a vaginal border, the height of the vaginal component should be measured and the posterior columns identified [1-3]. (Figure 1)

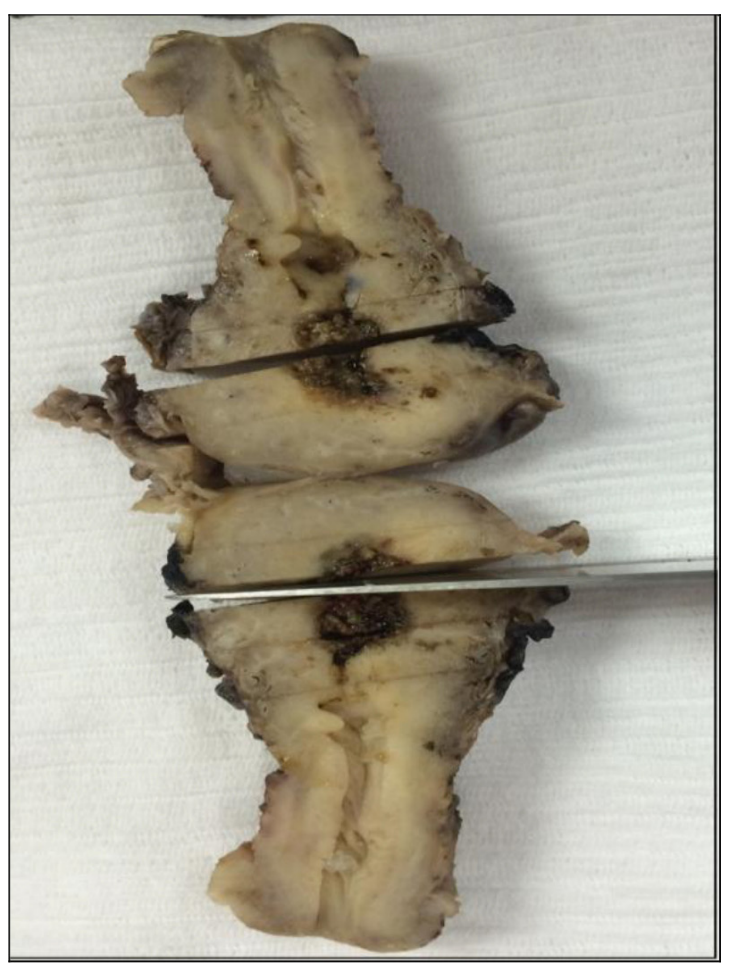

Figure 1 
A fundamental step is the examination of visualized lesions, detailing number, measurements (three dimensions in the neoplasm), outline, consistence, and color, relationship with adjacent and measured structures. Exception situations for exams of hysterectomies are represented by uteruses sent lacerated and fragmented in more than portions. The portions will be heavy in set; and measurements will be performed on the largest and smallest portions in the three dimensions. However, the approach to the neoplasm is impaired, especially with respect to the resection margins. The pathologist can make this reference in the final report, justifying, like this, the impossibility assessing the margins of surgical resection.

\section{Endometrial Adenocarcinoma}

Endometrial cancer is biologically and histologically diverse, being divided in two types: low-grade estrogen-dependent (well or moderately differentiated); frequently associated toendometrial hyperplasia; and type II which occurs more frequently after menopause has a high histological grade, as the serous carcinoma and clear cell carcinoma subtypes [3-5].

Usually, adenocarcinomas are located in the high and medium body. In some cases, they originate in the lowest segment of the uterine body. Often, in the macroscopic examination, they are dominant masses. They can be diffuse and superficial in the endometrial cavity. Many times, they extend to the internal or external half of the myometrium or to the serosa. It is important to assess the extension to the endocervical cavity and establish whether it is superficial or deep in the stroma $[3,5]$.

Therefore, specimens of the tumor with myometrium and painted serosa, the endometrium endocervical canal transition (isthmus) and the endocervix are collected for histology $[1,3]$. (Figure 2)

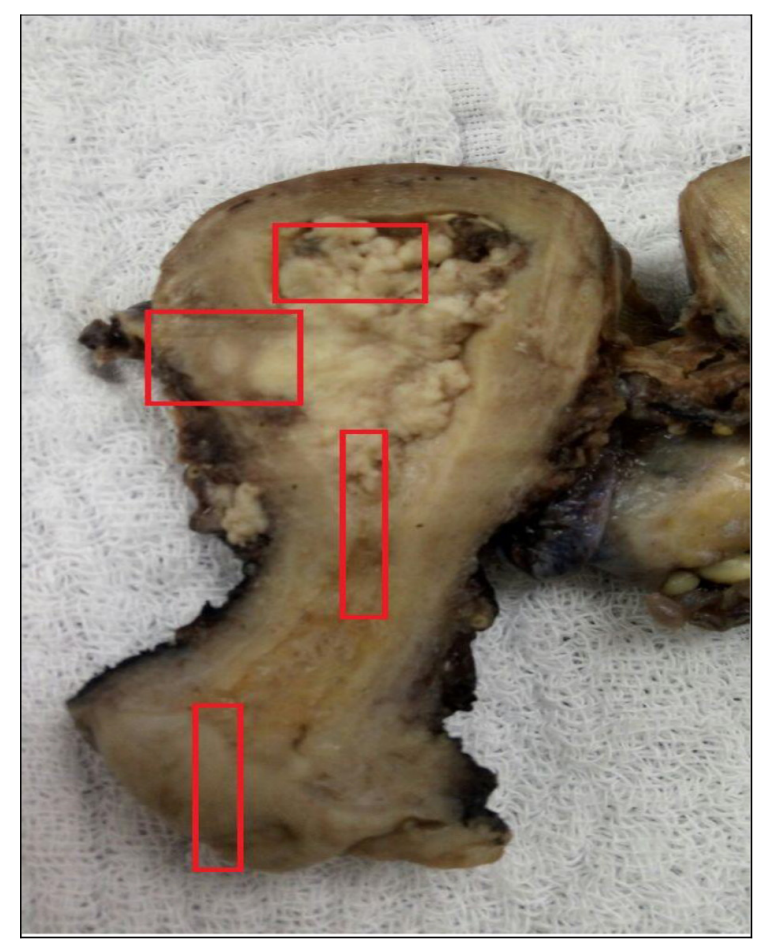

Figure 2
Each selected slice will be embedded in blocks, producing slides for microscopy and the final report in agreement with the macroscopic examination approach, describing the type endometrial lesion, its location: body, bottom, isthmus, penetrating the endocervical canal. The measurements of the neoplasm are taken in the three dimensions. The depth of the invasion in the myometrium and serosa is assessed, as well as the invasion of the isthmus, endocervical canal and ectocervix $[1,2]$.

This sampling procedure is important for the anatomopathological staging of type I and II adenocarcinomas and malignant mixed Mullerian tumors [5].

In possession of these data, a surgeon will be allowed to define survival and complementary therapies.

\section{Invasive carcinoma of the cervix}

The invasive carcinoma of the cervix can be, predominantly, vegetating or exophytic, papillary, polypoid or endophytic. When the neoplasm surrounds the cervix of the uterus and penetrates the underlying stroma, it produces a barrel shaped cervix, which can be identified by direct palpation. It may reach parametric soft tissues. In advanced stages, it extendsby directly spreading to contiguous tissues, such as the bladder, ureters, and vagina [3-5].

Therefore, in hysterectomies due to invasive carcinoma of cervix, we proceed by defining the lips and quadrants of the cervix that are involved by the neoplasm and the type of lesion (ulcerated, vegetating, infiltrating). The measurements of the lesion includediameter, extension towards the endocervical canal and lesion in the stroma. The invasion of the endocervix and right and left parametria, as well as the invasion of the endometrial cavity and vaginal cuff when present, should be described $[1,2]$.

The following specimens are collected for histology: lesion (both lips) with ectocervix or vaginal cuff painted withink; tumor and endocervix; assessing the thickness of the lesion; right and left parametria painted with ink, endocervix, endometrium transition, myometrium and other secondary lesions that may be found in the uterine body [1,2,3]. (Figures 3 and 4)

\section{Uterine Cervix X conization}

Cone macroscopy: for a satisfactory final result, it is recommended that the surgeon use a repair (12 hours) site to position the piece (cone). A good understanding between pathologist and surgeon is necessary. The surgical ectocervical and endocervical margins are painted with ink and fixed with acetic acid. The piece will be opened during the repair, when present or randomly.

The best way to fix the material is to stretch it out on cork or styrofoam, using pins to secure it, favoring thus its fixation without retraction and with a better sample of the squamocolumnar junction section [2].

The macroscop ic examination begins by measuring the height and diameter of the cervix (amputation or cone). The ectocervical surface is evaluated for the presence of rosy, ulcerated areas, with vegetations, light brown areas of leukoplasia, and the measurements of these and 
the determination of the quadrant in which they are located should be reported, as well as the distance of the endocervical and ectocervical margins $[1,2,5,6]$.

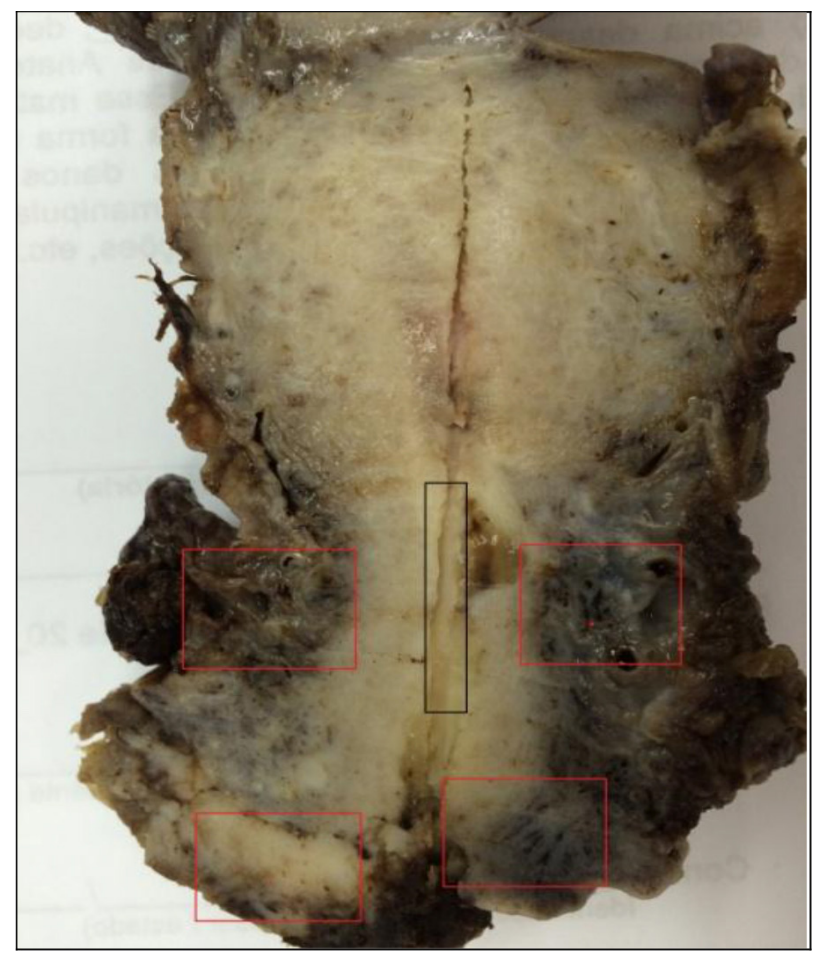

Figure 3

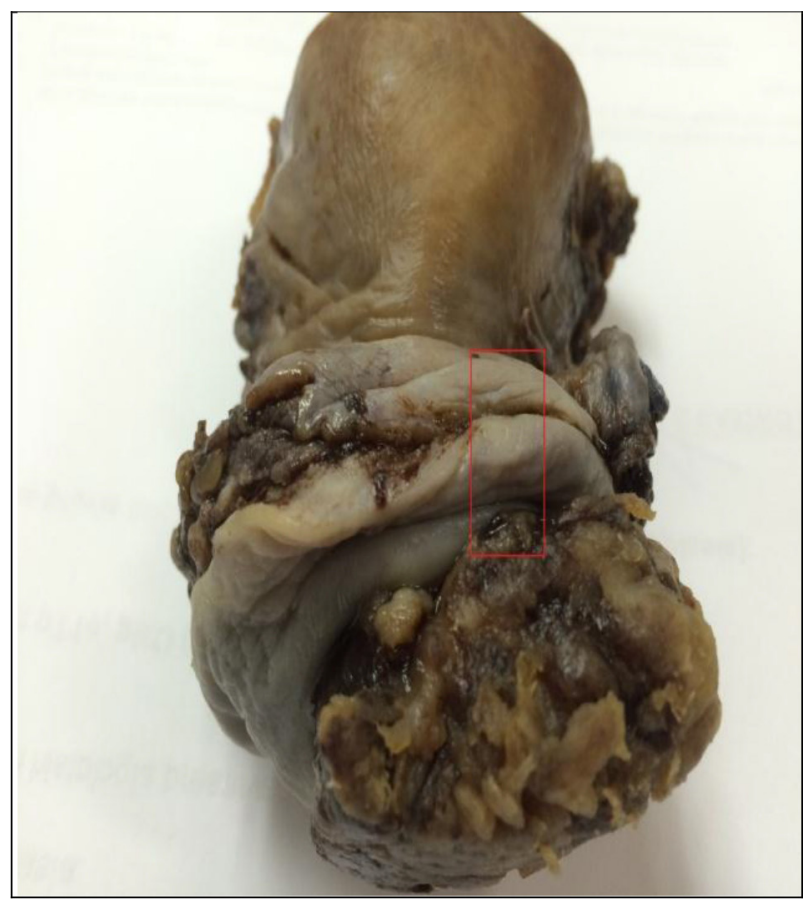

Figure 4

If the cone is higher than $2,0 \mathrm{~cm}$, it is possible to cut the endocervical margin and include it separately. On the other hand, when irregular and short slices are cut without separating the two margins, with the first, second, third and fourth quadrants being initially cut $[1,2]$.
The conization specimen should be included altogether, in radial (pizza) sections. ${ }^{1}$ Another option for cutting the cone is in parallel sections: cuts are performed in the anteroposterior direction. This type of cut is more difficult to be done when the cone cannot be previously stretched out and is already fixed [1].

\section{Generalities of macroscopy of the uterine tubes}

Neoplasms are rare, but we cannot forget them during the macroscopic examination. The carcinoma of the tubes usually occurs in their ampulla or fimbriae, the right tube being more affected than the left one. Macroscopically, in the lesion area, the tube is round, of showing a fusiform or hotdog shape. The surface is reddened, lumpy, with necrosis and blood content, or a raspberry color. The fimbriae can be closed, if the neoplasm spreads, the ovary and the uterus can be adhered, forming a mass. Cutting sections from the lesion (surface painted with ink) area, from the three portions of the tube, including the uterine ostium, is crucial. The full inclusion of the fimbriae is recommended. The tubal canal should be resected from the isthmus to the ostium.

\section{Ovaries}

\section{Macroscopy of the Ovaries}

In the macroscopy of the ovary, the presence of cystic, solid or solid-cystic lesions should be established. The first step consists of weighing the piece before opening it and measuring its three dimensions (including thickness and diameter) [1,2].

Subsequently, the configuration of the ovaries is observed, and the tunica albuginea is assessed. This can be complete, or lacerated, showing vegetations and nodules. The surface can be flat, bossy, light brown, dark brown, hemorrhagic and translucent.

Ovaries with abscesses or torsion show a dark brown external hemorragic surface with necrosis foci and fibrin deposits. Tumoral ovaries with laceration show whitish tumor areas in the tunica albuginea. Endometriosis foci (with a hemorrhagic or cystic aspect) can be present.

When the ovary presents a solid lesion or an area of adherence to the uterine serosa, this transition area should be evaluated histologically to discard invasion of the uterus (serosa, myometrium).

The opening of the ovary should start from the free margin towards the hilum (largest diameter). The evaluation of the internal surface is an important stage, where we needed to describe: cystic lesions and type of content (serous, mucinous, chocolate, and hemorrhagic) [2]. If there is more than one cavity, the diameter of the largest cavity, specimen collection: it should be 1 section from the largest dimension of the tumor. A whole slice can be achieved along the larger axis and included in its entirety, the transition area between the ovary and the tube should be included [1-3]. (Figure 5)

In the intraoperative examination: weigh, measure, open and empty the cyst(s). Assess the content of the cysts and the internal surface. Sample solid areas, when presents and cut the material in the cryostat to prepare a slide. In mucinous tumors, the surgeon should 
always be alerted about the possibility of finding a neoplasm in the subsequent examination in paraffin. The imprint exam can be used.

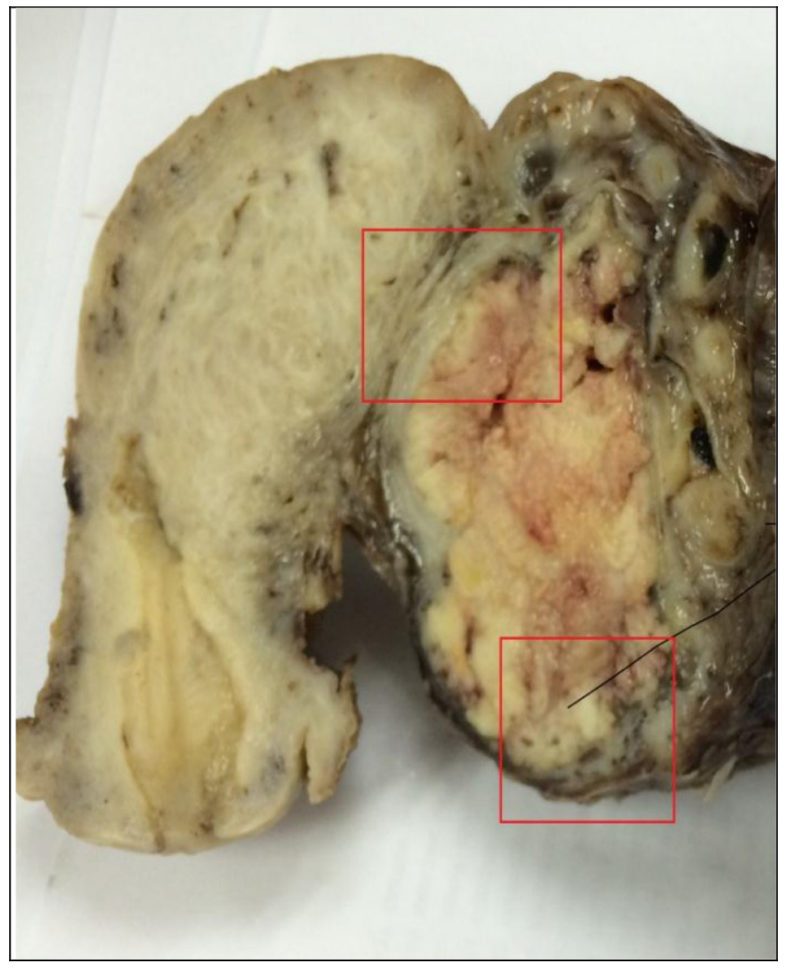

Figure 5

\section{Generalities of the macroscopy of the ovaries}

Ovarian teratomas-presence of hair, cartilage, fatty tissue, teeth, pasty and yellowish contents. The wall is fibrous, The collection of specimens should be satisfactory and comprehensive due to the possibility of detecting an immature component.

Mucinous cysts multilocular, bulky cystic masses, with gelatinous content. Malignant forms present solid areas, necrosis and nodules in the capsule.

Unilocular or multilocular serous cysts: the internal surface is flat and the content is serous. Malignant forms contain internal or external whitish papillary outgrowths, with necrosis areas and hemorrhage. The wall thickness is irregular, containing solid areas. Firm whitish nodules can be observed in the capsule.

Cystoadenofibroma-tumors with a cystic internal surface and usually serous content. The internal surface shows light brown papillary outgrowths.

\section{Macroscopy of the Vagina}

The primary carcinoma of the vagina is rare. More frequently, we find in macroscopy lesions due to invasion of uterine cervix tumors, or relapses of uterine cervix tumors as yolk sac tumors; or also red nodular lesions that correspond to pyogenic granulomas, or papillomatous lesions of viral origin [3-5]

Resection specimens can be sent and, therefore, we should paint the section (opposed to the mucous membrane) surface with ink.
If the piece is marked with a suture by the surgeon, demarcating its margins, its sections should be individualized, included in cassettes separately and identified.

\section{Conclusion}

People have been increasingly talking about interdisciplinary and multidisciplinary. Teamwork and good communication are key points for patient management. The surgeon, gynecologist, pathologist, oncologist, radiotherapists and depending on the type of neoplasm, other specialists, such as nutritionists, speech therapists, physiotherapists, nurses, psychologists, among others, meet the full set of requirements for a good performance and maintenance of the quality of life of cancer patients.

In this sense, we deemed important to describe the macroscopic examination of the female internal genital system so that the final report can be better understood by readers. All microscopy items refer to the slides prepared in agreement with the findings of the macroscopic examination, and staging will depend on these procedures.

\section{References}

1. Bacchi CE, Mello Cra, Franco M, Neto RA (Ed.)manual de padronização de laudos Histopatológicos. Sociedade Brasileira de Patologia, 4 ed. Editora Manole Ltda, são Paulo.

2. Becker, P. Manual de patologia cirúrgica. Guanabara Koogan, Rio de Janeiro, 1977.

3. Rosai, J. Ackermans Surgical Pathology, $10^{\text {th }}$ ed. Mosby, St. Louis, 2011.

4. Fletcher CD. Diagnostic Histopathology of Tumors. Churchill Livingstone, London, 2007.

5. Kumar, Vinay;ABBAS, Abulk.;Aster, Jon C.Robbins Patologia Básica.9ed Rio de Janeiro:Elsevier, 2013.910p.Tradução de Robbins basic Pathology.ISBN 9788535262940

6. Sobotta, Atlas de Anatomia Humana. Vol. 1 e 2. $22^{\mathrm{a}}$ Ed. Rio de Janeiro. Guanabara Koogan, 2002.

\section{Citation:}

Josenel Maria Barcelos Marçal, Rafael Nazário Bringhentti, Rita de Cássia Sant' Anna Alves, Adriana Vial Roehe, Fernanda Correa Pinto, Judite Tais Silveira Fagundes, Péttala Rigon, Shala Wesner Martins and Giuseppe Dick Bonato (2019) Understanding the Macroscopy of Malignant Lesions of the Female Internal Genital Tract. Integr Gyn Obstet $J$ Volume 2(1): 1-4. 